\title{
Veiller, accompagner : traces reverdyennes dans Avant la règle (2014), Carnets à bruire (2014) et Un versant l'autre (2019)
}

Les énoncés de Pierre Reverdy, d'André du Bouchet et d'Esther Tellermann peuvent nous surprendre, par eux-mêmes ainsi que par leurs ressemblances : le ciel qui nous « soutient», où se laver les yeux « tous les matins $»^{1} ;$ 《le regard, / écharde qui éclaire $»^{2} ;$ « Distance [...] surcroît de paroles » $(C B$ 92) ; « dans la syllabe pleut / la lueur» $(A R 21)$; « en mots simples advenir » (UV $69) .{ }^{3}$ Chacune de ces citations met en évidence la manière dont on avance poétiquement dans le monde pour mieux être, par le regard et par les « mots simples » qui donnent à voir. Le lecteur de ces trois poètes apprend à accueillir le réel tout en acceptant que celui-ci soit troué. Quelques mots rythmés, qui s'accordent au va-et-vient de la respiration et aux sauts et percées de l'appareil perceptif, peuvent tisser des liens entre soi et l'Autre, l'acte de voir et ce qui est $\mathrm{vu}$, l'ici et maintenant et l'à-venir presque à notre portée. On « embrasse [...] l'univers $»^{4}$ avec reconnaissance, sans oublier nos manques et insuffisances. Il reste toujours quelque chose à revoir ou à refaire, le devenir à revisiter.

L'analyse suivante se propose de montrer de nouveau des influences qui façonnent le regard tellermannien sur la parole, la profondeur et l'éclat du dire dans ses recueils au plan du tissu allusif, en mettant en lumière des liens de parenté entre Reverdy et Tellermann. Au premier abord, ces liens semblent indirects, dans la mesure où Du Bouchet sert d'intermédiaire. C'est à lui par exemple qu'est dédié Carnets à bruire, recueil que Tellermann a écrit en lisant les carnets de son aîné et où, d'une manière singulièrement laconique, il est plus souvent question de problématiques dubouchettiennes telles que le « lieu / grand ouvert » qui devient visible lorsqu'on avance « dans / l'incendie » $(c B$ $7,48)$ que de fugaces drames humains face à un réel plein d'ombres. Or, les

1 Pierre Reverdy, «Horizon », Quelques poèmes [1916], QEuvres complètes I, éd. Étienne-Alain Hubert, Paris : Flammarion, 2010, p. 62-74 (71; cf. 1140-41).

2 André du Bouchet, Carnet 2, op. cit., p. 100.

3 Les poèmes de Carnets à bruire sont « dédiés à André du Bouchet» ( $C B$ 101) et composés à partir de ses recueils Carnet, Carnet 2 et Annotations sur l'espace non datées (Carnet 3 ) (Saint Clément : Fata Morgana, 1994, 1998 et 2000). LT suggère d'autres liens à André du Bouchet : la vignette de couverture est de son fils, Gilles du Bouchet.

4 Michel Collot, « Reverdy selon Du Bouchet », op. cit., p. 100.

(C) AARON PREVOTS, 2022 | DOI:10.1163/9789004499683_009

This is an open access chapter distributed under the terms of the CC BY-NC-ND 4.0 license. 
liens entre Reverdy et Tellermann sont très directs, profonds même. ${ }^{5}$ Elle s'inspire également de lui, dans la mesure où les recueils récents de Tellermann reprennent certains motifs reverdyens pour commenter la condition humaine et affinent le dire pour épaissir musicalement le dit. Parmi les emprunts à Celan ou à Perse se trouvent d'autres approches et structures que l'œil attentif peut discerner, même si les contours de celles-ci tendent à s'effacer dans le cadre d'une épopée singulière, du Livre-poème ${ }^{6}$ que tisse Tellermann au fil des ans. Des ouvrages de Guerre extrême à Éternité à coudre, toutes influences confondues, traitent de préoccupations qui perdurent. Ils privilégient l'ellipse, l'instantané et des gestes vers l'Autre à peu près comme le ferait Reverdy, tout en s'inspirant en même temps de Du Bouchet.

Une lecture attentive d'Avant la règle, Carnets à bruire et Un versant l'autre montrera, surtout par le biais de Du Bouchet, de nombreux points de convergence entre Reverdy et Tellermann : une traversée physique et spirituelle du réel ; une découverte constante de l'épaisseur du monde ; une commémoration sous-jacente des morts et de la mort ; un rapprochement de « réalités plus ou moins éloignées » ${ }^{7}$ présenté comme socle de l'acte poétique ; une recherche de la lumière, de ce qui dans le monde extérieur nous sert de promesse chaleureuse et sensuelle; le recours à la parole poétique comme prière qui nous relie aux morts; et la création de paysages sonores, où éclot en permanence l'émotion qui sous-tend ces procédés. Cette liste n'est pas exhaustive. Une lecture en miroir de leurs ouvres dépasserait notre cadre. Il importe avant tout de voir comment Tellermann veille dans un monde trouble sur le langage et le réel en inscrivant dans ses recueils des aspects de la poétique reverdyenne ou dubouchettienne.

5 Correspondance avec l'auteur, 22 mars 2021, remarque intégrée avec son aimable autorisation.

6 Nous avons parlé par moments d'un livre-poème $(E R, L T, E C)$. Il est possible de mettre une majuscule au mot « Livre », car il s'agit pour Tellermann de se tendre dans son écriture « vers le Livre », d'ajouter aux écrits qui constituent l'héritage culturel commun à tous. C'est un fil conducteur lié à « [s] judéité » (correspondance avec l'auteur, 20 mars 2021, remarques intégrées avec son aimable autorisation). On peut identifiér son projet poétique non seulement à la fable ou au mythe, mais aussi à l'énigme de la parole diasporique au sens le plus large possible. Cf. nos remarques dans le chapitre 2 sur Saint-John Perse, ainsi que Jean-Claude Poizat, «Entretien avec Daniel Sibony », Le Philosophoire 31 (2009/1), p. 211-28 (216), <cairn.info>, où celui-ci explique que l'identité juive est « la transmission du manque qu'elle comporte, du manque-à-être et du désir de le surmonter grâce à la transmission elle-même », transmission qui assume en effet « sa propre faille et cet aspect entre-deux, précaire ou bancal, [...] compt[ant] sur son échec pour rebondir ».

7 Pierre Reverdy, Nord-Sud 13 [mars 1918], CEuvres complètes I, op. cit., p. 495-99 (495). 
Il convient dans un premier temps de comprendre à travers Michel Collot « la lecture qu'André du Bouchet a pu faire de la poésie de Reverdy ».8 Ainsi verra-t-on comment nos trois poètes prennent le relais l'un de l'autre, privilégiant des univers symboliques semblables. D'abord, il y a le fait que l'héritage concret de l'entre-deux-guerres pour eux tous, c'est le sentiment que le monde va de plus en plus mal, et la conviction que la poésie sert à le soigner, à le réparer, à trouver les mots capables de « restaurer par l'écriture » notre « relation perdue » avec lui. ${ }^{9}$ Le fond de leurs poétiques respectives est une distance prise vis-à-vis du discours ambiant et le souci de créer un autre discours qui lui serve de contrepoids plus ample, vrai, généreux. Ils ressentent surtout cette opposition, cette dimension relationnelle, par exemple lorsque leurs vers sont brisés, ciselés, épurés, pleins de regards brefs, d'hésitations, de lumières étincelantes, d'ombres anguleuses, de souffles tantôt amples, tantôt cristallisés. Par ailleurs, leurs démarches s'ancrent dans des souffrances personnelles liées à l'Histoire, qu'il s'agisse du service militaire et de l'Occupation chez Reverdy, de l'exil d'André du Bouchet avec sa sœur et ses parents pendant huit ans aux États-Unis suite à des persécutions antisémites ou du cataclysme de la Deuxième Guerre mondiale vécu à distance par Tellermann..$^{10}$ Il ne faut pas oublier l'« éboulement $\gg^{11}$ dont parle Du Bouchet comme arrière-plan de la poésie contemporaine et comme présence dans l'univers reverdyen, où les êtres et les choses constitutifs du monde matériel passent et tombent, se cachent et se perdent. Selon Du Bouchet, il y a une « violence [...] toujours à l'œuvre sous nos yeux », violence socioculturelle et historique où se détermine « un rapport d'affrontement » avec le langage. Ces tensions mènent à une ouverture intersubjective, à la « fraîcheur » qui a lieu lorsque le langage « ne se referme pas sur soi ». ${ }^{12} \mathrm{La}$ tendresse de Tellermann émane de ces abîmes. ${ }^{13}$

8 Michel Collot, « Reverdy selon Du Bouchet », op. cit., p. 94.

$9 \quad$ Ibid., p. 95. Voir aussi Michel Collot, « La "langue-peinture" : Du Bouchet, Jaccottet, Bonnefoy », Esprit 213.7 (juil. 1995), p. 138-41 (139).

10 Mary Ann Caws, ed., Pierre Reverdy, trans. John Ashbery et al., New York : NYRB, 2013, p. xi-xxii ; André du Bouchet, Aveuglante ou banale : essais sur la poésie, 1949-1959, éd. Clément Layet et François Tison, Paris : Bruit du temps, 2011, p. 10 ; André du Bouchet, Entretiens d'André du Bouchet avec Alain Veinstein (1979-200o), op. cit., p. 99-100 ; André du Bouchet, Openwork: Poetry and Prose, op. cit., p. xiii-xviii ; Clément Layet, «"La question du soleil" : André du Bouchet et la question politique », op. cit., p. 99. Du Bouchet a des origines juives russes du côté maternel.

André du Bouchet, Entretiens d'André du Bouchet avec Alain Veinstein (1979-200o), op. cit., p. 28, p. 115 .

Voir aussi $U O$ et $P V$. 
Les ressemblances les plus fortes entre Reverdy et Du Bouchet que soulève Collot se situent au niveau du ton, des motifs, des rythmes et de la transitivité, notamment du regard tourné vers autrui et le monde. À vrai dire, comme le signale Collot, il faudrait parler plutôt de réflexes poétiques en début de carrière, puis de commentaires en tant que critique, et enfin d'une identification et d'une compréhension - ainsi que d'une amitié entre le maître et son disciple - qui permettent à Du Bouchet de passer outre à ses influences, de «trouver sa voie/x propre ${ }^{14}$ Après s'être défait du «mimétisme $»^{15}$ de ses débuts, Du Bouchet se distingue selon Collot en analysant avec justesse les poèmes de Reverdy; en développant avec intelligence un projet d'une même envergure ; en devenant lui-même une sorte d'«homme-paysage », ${ }^{16}$ poétiquement en osmose avec le jaillissement du réel ; et, lors de la publication en 1992 du livre consacré à Reverdy Matière de l'interlocuteur, en devenant cet « interlocuteur » qui n'hésite ni à réécrire et condenser ses textes analytiques, ni à y insérer en italique des fragments de poèmes de Reverdy pour qu'il y ait « dialogu[e] d'égal à égal $»{ }^{17}$ C'est cette dernière inflexion - au-delà d'images ou de motifs particuliers - qui compte le plus pour Collot : « une approche inédite du réel », ${ }^{18} \mathrm{et}$ qui a une "signification métapoétique ${ }^{19}$ dans la mesure où elle oblige tout poète à se tourner avant tout « vers le monde.$^{20}$

Tellermann profite de certains liens à Du Bouchet pour poursuivre sa réflexion sur l'écriture. Son regard sur lui en tant que critique elle-même amplifie ce qu'elle est déjà en train de faire comme poète, à savoir «accueillir le monde », se tourner vers l'espace du dehors et du dedans, explorer « la spatialité sonore du poème » où s'élève l'éclat sacré de «ce qui reste à dire » $(N N$ 23,26 ). Ce qu'elle fait comme poète est en soi reverdyen, dans le sens où elle élabore un art relativement «autonom[e] » insufflé de sa propre «émotion poétique », n'abordant que de biais, de loin, les « spectacles de la nature ». ${ }^{21}$

Poursuivons, comme le ferait Collot, en mettant l'accent sur le «lyrisme de la réalité $»^{22}$ reverdyen que l'on peut remarquer chez Tellermann. Creusons les aspects cités plus haut qui resurgissent dans Avant la règle, Carnets à bruire et

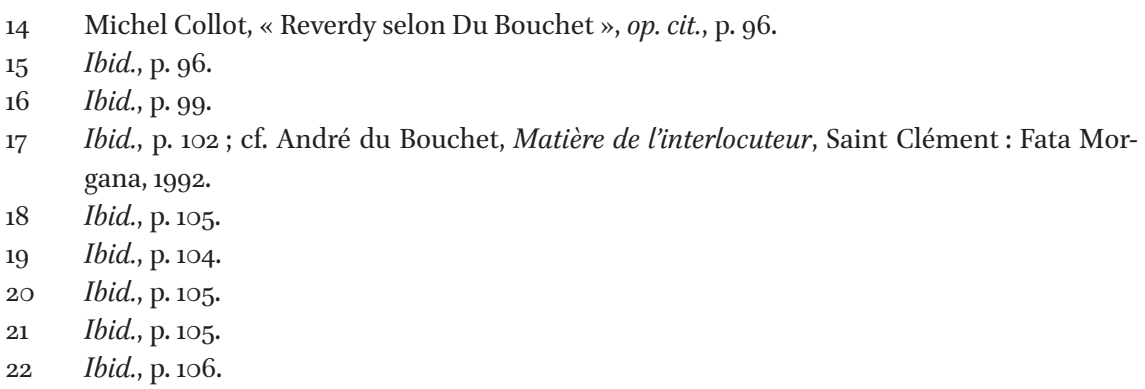


Un versant l'autre. Commençons par la traversée du réel dans Avant la règle, les coupures visuelles et syntaxiques qui suggèrent un va-et-vient « dansant » $(A R$ 15), ainsi que les visées identitaires quant à la construction de suites. À la différence de Du Bouchet, Tellermann emprunte à Reverdy certains procédés qui sont peu présents dans les carnets, comme si finalement les influences avaient sauté une génération, évolué comme elle le dirait selon une autre «structure psychique $»{ }^{23}$ En tant que livre de petit format - $11 \times 16 \mathrm{~cm}$ - qui nous met à la recherche d'un «lieu d'innocence » $(22,34 ; \mathrm{cf} .20,39)$, Avant la règle se rapproche des plaquettes et livres d'artistes de Reverdy, ${ }^{24}$ reflétant un désir de faire de l'art un tel lieu d'innocence, le cœur même du « vertige d'une spiritualité », 25 un horizon où ciel et terre s'unissent. Par rapport à cette production artistique du début du vingtième siècle, la disposition typographique des poèmes de Tellermann semble s'inspirer de Reverdy, qui met des alinéas à des moments inattendus mais bien mesurés et prête ainsi au poème une cadence légèrement saccadée, comme s'il restait toujours à l'écoute du vent qui « conti$\mathrm{nu}[\mathrm{e}]$ sa ronde $» .{ }^{26}$ À ce dernier propos, Avant la règle fait partie d'un diptyque avec Un point fixe, livre de deuil publié lui aussi dans la collection « cendrier du voyage » le 21 mars 2014, où elle cherche des « lueurs afin que / deviennent / les horizons » et voit « les sables / / dans les soleils» (UP 30). Autrement dit, Avant la règle et Un point fixe sont conçus comme des boussoles pour des lecteurs ayant soif d'une mélancolie reverdyenne, des mêmes échardes de motifs qui percent lâme, mais qui veulent néanmoins boire une lumière élémentaire qui les soulage et rassure, qui leur rappelle les cycles du monde extérieur et donc « ouvr[e] / un infini » (UP 37), celui du regard libre de trouver les lieux physiques et psychiques où naît le poème.

Quelque chose de l'ordre du sacré nous frappe immédiatement dans Avant la règle. Le registre est soutenu, mais prend aussi les inflexions d'un échange familier. Il est question à la fois d'un «temple», de «murs aveugles» et de « tissus » (AR 7). On se trouve « aux croisements des principes et des bassins » (7), de l'abstrait et du concret, du présent de la découverte et des « rites et sacrifices » (17) d'un quotidien épique et lointain, lequel s'avère plein de « sédiments » et d'« histoires » (10). La verticalité du premier texte et ses vers de un à quatre mots font penser à une inscription qui se trouverait près de ce temple,

\footnotetext{
23 Aaron Prevots, « Entretien avec Esther Tellermann », op. cit., p. 108.

24 Pierre Reverdy, Fac-similés des premières éditions: La Lucarne ovale [1916], Quelques poèmes [1916], Les Ardoises du toit [1918], CEuvres complètes I, op. cit., p. 1043-1233.

25 Étienne-Alain Hubert, dans Pierre Reverdy, QEuvres complètes I, op. cit., p. 1279.

26 Pierre Reverdy, «Voyages sans fin», Sources du vent [1929], CEuvres complètes II, éd. Étienne-Alain Hubert, Paris : Flammarion, 2010, p. 15 o.
} 
et l'emploi du pronom sujet « on » renforce le fait qu'il s'agit de collectivités : «C'était avant la règle / on construisait / des murs aveugles / on tendait des tissus » (7). Notons au passage que cette verticalité nous met « debout / contre les / chiffres » $\left(\begin{array}{ll}C B & 16\end{array}\right)$, prêts à traverser des contrées d'« avant la règle », c'est-à-dire d'avant les concepts et idéologies qui gèrent dangereusement notre civilisation actuelle et rendent la « fraîcheur communicative ${ }^{27}$ difficile d'accès.

Le «lyrisme décentré ${ }^{28}$ des textes dans Avant la règle esquisse de façon gnomique des gestes vers « [q]uelque chose [qui] ne sera / rapporté » $(A R 7)$, interroge le réel sans répondre aux questions ouvertes qui sont implicitement posées. Le dire assez hiératique - « tu es / visible / je suis déracinée / dehors » (9) - s'accentue sur chaque page lorsque des alinéas et des mots ainsi isolés frappent le regard et l'oreille. On peut constater un mouvement en avant vers un Tu qui est autant ce dehors qu'un interlocuteur bien aimé : «j'ai vu / les éclats bleus / des quartz / ta face / était le chiffre / et l'alliance / une orgue / / sur les épitaphes » (53). Comme c'est le cas pour Reverdy, l'épaisseur du monde se crée à partir de plusieurs spécificités du texte poétique : ce mouvement en avant; ces «éclats » du monde extérieur qui surgissent au sein du poème ; cette « alliance » entre soi et l'Autre, le moi et le monde ; la vision et les gestes qui instaurent ces liens intersubjectifs; et la vie et la mort vues comme une unité, une continuité, des parties d'un tout qui nous dépasse. Le cheminement en avant du lecteur, sa traversée du livre, l'emporte sur la cristallisation d'un sens. Le «lieu d'innocence» $(22,34)$ que l'on parcourt se situe dans le réel, mais, comme chez Reverdy, est aussi son propre lieu, son propre réel, façonné d'éléments épars du présent et du passé, d'un ici et d'un ailleurs. Tellermann reprend en termes plutôt autoréférentiels ${ }^{29}$ des problématiques suggérées par son aîné : « un jet de pierres brûle / le sac à paroles » (7); « chant ou frontière / furent le signe » (11); « une douleur sur l'arête / d'une lettre-étincelle» (27); « je t'effeuille / nous ne serons pas / tout à fait » (33); « sous les chambres est / l'asile / dans le mutisme de / l'appel » (45). L'épaisseur du monde est aussi la tension sismique qui s'opère dans le poème. La voie poétique nous initie à un savoir intuitif, par lequel le «chant » est en lui-même « signe », «l'arête » des mots l'appel d'un incendie qui guérit, l'espace d'où l'on regarde - «les chambres » - un point d'appui où l'on n'est qu'en apparence muet, où le silence peut s'épandre. Lire Tellermann en gardant en esprit Reverdy, c'est se réjouir de la contiguïté entre la littérature d'époques diverses. Condenser le dire comme

\footnotetext{
27 André du Bouchet, Entretiens d'André du Bouchet avec Alain Veinstein (1979-200o), op. cit., p. 116.

28 Alain Cressan, « Esther Tellermann : Avant la règle/Un point fixe », op. cit.

29 Cf. Michel Collot, « Reverdy selon Du Bouchet», op. cit., p. 105.
} 
elle le fait peut déconcerter, mais nous dirige d'autant mieux vers le réel lorsqu'on comprend ce qui s'y inscrit en filigrane.

Découvrons d'autres points de convergence entre Reverdy et Tellermann en examinant Carnets à bruire, dont le trait le plus saillant est le fait que Tellermann accompagne Du Bouchet, le regarde, l'écoute, lui tend la main ( $\angle B 18$, 24), marche « sous [son] épaisseur » (26). En parlant pour lui, en « ébruit[ant] [son] / carnet / dans la bouche » (30), elle parle aussi - en creux, chuchotant au lecteur averti - pour Reverdy. Comment procéder en tant que critique ? Les palimpsestes reverdyens seraient nombreux. Contentons-nous de souligner quelques motifs et procédés qui font s'emboîter les textes de nos trois auteurs. Il y a par exemple dans Carnets à bruire un contact insistant, à la fois retenu et réjouissant, avec le dehors. Le cadre métrique est sobre, fait généralement de dissyllabes, de trisyllabes, de tétrasyllabes et de pentasyllabes. Les énoncés sont troués, juxtaposés. Or, le fond en est lumineux, plein d'appels profonds, ruisselant de haut en bas de la page. Tellermann évoque d'emblée des lieux et espaces dubouchettiens. Les mots sont faits pour montrer « les lointains » (85), « écaill[er] la surface » (94), glaner des « parcelles / de durée » qui « ouvrent le passage où / sommes en suspens » (95). Il peut y avoir de l'« inquiétude » (62) comme chez Reverdy, l'«à vif » (98) peut peser et faire «tomb[er]» $(17,18,26$, 55), mais le recours fréquent à l'idée de «forces ramassées / dans le chant / à portée de main » $(23 ; \mathrm{cf} .28,29,34,44,80,98)$ met en relief l'épanouissement du monde sensible que l'on ressent à même la chair et l'âme grâce au poème. Que l'on songe aux recueils ou aux proses, à la forme ou au fond, les écrits de Reverdy se font sentir dans cet hommage à Du Bouchet.

D'autres mots qu'emploie Tellermann sont également des reprises de ce que font ses deux aînés quant à l'écriture comme champ tellurique où l'on avance $(9,10,92)$, nomme (13) et moissonne $(31,32,77 ; \mathrm{cf}$. 80). N'oublions pas les éléments : l'eau, l'air, le feu, la terre, chacun moins une réalité fixe que ce que le texte poétique fait voir, couler ou bouger, par exemple dans le cas des « ciels arrachés » (9). Des vers qui peuvent nous rappeler le début d'Avant la règle en ce qui concerne l'abstrait et le concret qui se croisent - « Routes [...] tournaient / les espaces / dans les axiomes et les / orages / restituent / la respiration » (10) - relient de manière reverdyenne le dedans et le dehors, les « orages » (10) de la vie intérieure et le discours normatif des «axiomes » (10), la spiritualité de l'acte poétique et le «réel désiré qui manque ».30 Tellermann accentue la spiritualité lors de sa relecture des carnets, en en raccourcissant les rythmes et en en « rassembl[ant]» (10) le contenu pour le faire entendre comme un 
« murmure » (89), l'une des « langues / murmurées » (20) par le monde extérieur. Songeant peut-être à Reverdy et parlant à distance de Du Bouchet, elle s'occupe moins de leurs observations du réel que de leur nomination des aspects clés du processus poétique. Ainsi en est-il des mains et des paumes qui cueillent les signes, ${ }^{31}$ comme du «soleil » et de la «nuit» $(10,20,27)$ que les noms peignent métaphoriquement. Tout en suivant les traces des auteurs qui l'ont précédée, par exemple les «sillons » $(74,83,94)$ et «ornières » (24, $80)$ éblouissants de l'« encre » $(14,28,58)$ ou la « flaque de lumière » (19), elle ajoute des touches personnelles afin d'aviver le dire poétique, d'en faire une synthèse, de scruter nos espaces intérieurs personnels et collectifs. Du Bouchet disant pendant l'hiver 1952 son besoin de vivre « la mort», de « $\operatorname{racl}[\mathrm{er}]$ la terre avec [s] on carnet » pour « respirer » ce qu'il voit, ${ }^{32}$ peut ainsi se traduire par des vers où Tellermann situe le dire sur un plan aussi culturel et civilisationnel que poétique: «Je dis / les Dieux nus / valses éteintes et / vertiges / pierres écorchées » (19); « barques ne faisaient plus / le lien des / Empires» (48; cf. 39, 52). On peut observer d'ailleurs combien les intertextes s'entrecroisent aux plans lexique et formel, en se rappelant Celan, Du Bouchet, Perse, Reverdy, les vertiges et les vers courts des uns ou la barque poétique peu amarrée des autres.

Revenons au sacré. Il s'inscrit dans ces Carnets à bruire par le biais de mots polysémiques, dont certains sont lourds de sens à force de figurer dans la poésie des dernières décennies, par exemple «épaisseur », « matière », «braise », «terre », «poussière » (7-13). En outre, prendre en compte un «tumulte» à maittriser $(78),{ }^{33}$ se tourner vers ses pairs et leurs œuvres pour le soigner, c'est déjà s'engager dans les voies du sacré. Trouver des moyens de dire la «braise » (34) et le « souffle» $(18,35,46,74)$ qui nous animent, c'est rapprocher des « réalités plus ou moins éloignées ${ }^{34}$ pour mieux saisir l'insaisissable et atteindre l'Autre. Ce n'est ni un jeu, ni la simple mise en branle de l'inconscient ou du monde extérieur, mais encore une autre façon de traverser physiquement et spirituellement le réel. Comme l'explique Du Bouchet, il s'agit d'«activer» le sens pour le mettre toujours « au futur», le rendre «mobile» et «mouvant», recommencer avec chaque texte à créer «le mouvement d'une relation instaurée », à mettre en place de vrais « échange $[\mathrm{s}]$ ». ${ }^{35}$ Rencontrer l'épaisseur du

\footnotetext{
31 Un passage sur la main comme « résumé de l'homme » est intéressant à ce propos (ibid., p. $835^{-36)}$.

32 André du Bouchet, Carnet, op. cit., p. 45, p. 47 et p. 5 o.

33 Cf. Michel Collot, « Reverdy selon Du Bouchet», op. cit., p. 98.

34 Pierre Reverdy, Nord-Sud 13 [mars 1918], CEuvres complètes I, op. cit., p. 495-99 (495).

35 André du Bouchet, Entretiens d'André du Bouchet avec Alain Veinstein (1979-2000), op. cit., p. 26 , p. 27, p. 86 .
} 
réel au moyen de l'écrit comme le font Reverdy, Du Bouchet et Tellermann, c'est donc vivre une relation bénéfique parce que continuellement réinventée.

Quelques vers en particulier dans Carnets à bruire soulèvent des enjeux identitaires mis en acte par les formules : «j'écris comme / je marche / devant et après / soi » $(20)$. Les avancées provisoires qui s'incarnent là sont des traces reverdyennes par excellence, des reflets de «lâme trouble $»^{36}$ de tout poète. Le vocabulaire assez abstrait de l'altérité que choisissent Du Bouchet et Tellermann s'adapte mieux à leurs discours poétiques respectifs, ${ }^{37}$ mais fait écho néanmoins au flottement identitaire que dépeint Reverdy quant à l'individu, aux êtres et aux choses qui nous entourent. Signalons d'ailleurs « la fragilité et la pauvreté $»^{38}$ dont Tellermann nimbe son hommage. Dans ces textes, seule la simplicité est de mise. La page de couverture « reproduit à l'envers le premier poème du recueil, comme si l'encre encore humide avait marqué la page précédente », suggérant l'impossibilité de « résister au temps » ${ }^{39}$ et les carnets comme espace de découverte de soi. C'est ainsi que ce recueil trace des gestes vers le sacré. La locutrice tend des «[p] eaux de papier» sur nos «silences», pour que « s'ouvrent » les années et « reviennent les [...] fraîcheurs » $(44 ; \mathrm{cf}$. 98). Ce contact se veut calme, respectueux, frôlant l'indicible, un bruissement « cohéren[t]» (64) aux niveaux du temps et de l'espace vécu dans et par le langage.

Ce bruissement se fait sentir tout particulièrement dans Un versant l'autre. Les exemples y foisonnent de la langue comme tissu sonore, étoffe qui aide le sujet poétique à tendre la main vers l'Autre et à s'adonner à une prière prolongée. Comme un Reverdy réitérant des mots et des motifs, ${ }^{40}$ Tellermann y sculpte avec grand soin les paysages sonores, se sert de répétitions pour créer

36 Pierre Reverdy, Les Épaves du ciel [1924], CEuvres complètes I, op. cit., p. 1281.

37 Exemples du vocabulaire de l'altérité dans les carnets : «j'écris comme on marche - à l'aveuglette, même en plein jour / comme on va devant soi, sans songer même à marcher » (André du Bouchet, Carnet, op. cit., p. 53 [févr. 1953]) ; "points aveugles de ma poésie / c'est par eux que je veux voir» (André du Bouchet, Carnet, op. cit., p. 54 [févr. 1953]); « une parole [...] dans sa volute / toujours plus avant le révolu / où soi-même on peut par instants se trouver à découvert, / et, un peu plus loin alors, / à un centre qui se soustrait » (André du Bouchet, Carnet 2, op. cit., p. $3^{1}$ [1962]) ; « au défaut du discours / ce qu'alors un peu plus haut que soi, / comme éclat du sol, / la langue a pu aller sans dire » (André du Bouchet, Annotations sur l'espace non datées (Carnet 3), Saint Clément: Fata Morgana, 200o, p. 7 [1983-1999], l'auteur souligne). Exemple chez Reverdy : « Je voudrais être loin de moi » («Enfin », Plein verre [1940], Pierre Reverdy, OEuvres complètes II, op. cit., p. 333). Martine Créac'h, «Note de lecture: Esther Tellermann, Carnets à bruire et Nous ne sommes jamais assez poète », Littérature 181 (2016/1), p. 114-15 (114), <cairn.info>.

39 Ibid., p. 114.

40 Michel Collot, « Reverdy selon Du Bouchet », op. cit., p. 99. 
des présences, pour habiter la langue et en faire sa demeure, « l'arracher à l'emprise des conceptions institutionnalisées par l'usage $»_{.}^{41}$ Faire bruire le langage, c'est donc veiller sur soi, le réel et l'Autre. Par le geste poétique, on leur tend la main, « retrouv $[\mathrm{e}] »$ l'amour malgré les horizons élimés, les ciels usés, tout ce qui «se dérobe » à la quête du poète et « lépuise ».42 $\mathrm{Si} \mathrm{le} \mathrm{mot} \mathrm{doit} \mathrm{signifier}$ pour combler un manque, il n'en reste pas moins vrai que le poète apprend à créer un « contexte » où « l'esprit » saisit un sens au travers de chaque « note » qui se produit, qui « vibre » par rapport à celui-ci et fait donc s'imbriquer les plans sonore et sémantique. ${ }^{43}$ Par ailleurs, répéter autrement ce qui a été dit est, selon Reverdy et Du Bouchet, une façon de respirer, de tisser des liens entre le dedans et le dehors, voire d'atteindre l'« Au-delà » en cherchant au moyen de mots qui nous habitent « tout ce qui est en dehors de notre étroite peau ».44

Divers procédés font sentir ce bruissement. Comme nous avons pu le constater à propos de Terre exacte et de Contre l'épisode, Tellermann aime à réemployer des mots, des motifs, des vers, des situations dialogiques. À ces niveaux, on peut observer des parallèles directs entre les remarques de $\mathrm{Du}$ Bouchet à propos de la poésie de son aîné et la méthode tellermannienne : «la disposition des points d'appui n'est jamais acquise d'avance [dans ce monde] liquide, fuyant, limpide»; «les mots se détachent par groupes à contrecourant et se recomposent [...] dans le fil de la dispersion $\gg .{ }^{45}$ Allons voir dans Un versant l'autre, prenant en compte le ton de ce volume récent par rapport à l'œuvre tellermannienne des années 1980 - ses «moments de grâce » et ses «mystère[s] », la manière dont la trame du poème «laisse toujours entrevoir le souvenir d'un chant plus ancien ». ${ }^{46}$

Notons d'abord combien ce titre est un leitmotiv tellermannien. La dispersion des sons et du sens dans cette image et dans les quelques mots qui la constituent sert à nous faire puiser dans un passé mythique, à suggérer un ici et un ailleurs, à retisser le fil du temps où les villes semblent devenues « closes », « épuisé[es]» $(U V$ 7). Ces versants sont loin d'être des lieux ou objets précis. Le lecteur les visualise en esprit. Enlever un déterminant ou un pronom sujet

\footnotetext{
41 Michel Collot, « La "langue-peinture" : Du Bouchet, Jaccottet, Bonnefoy », op. cit., p. 139.

42 Pierre Reverdy, Cette émotion appelée poésie [1950], CEuvres complètes II, op. cit., p. 1282-94 (1291).

43 Pierre Reverdy, En vrac [1956], CEuvres complètes II, op. cit., p. 840.

44 Le Livre de mon bord [1930-1936], CEuvres complètes II, op. cit., p. 643-803 (659).

45 André du Bouchet, «Envergure de Reverdy » [1951], Aveuglante ou banale : essais sur la poésie, 1949-1959, op. cit., p. 47-63 (52).

46 [Sans nom / Note de lecture non signée], «Un versant l'autre, Esther Tellermann », A.L.I. [Association lacanienne internationale], 9 sept. 2019, <freud-lacan.com>.
} 
l'encourage dans cette démarche ${ }^{47}:$ "Villes étaient / closes / avions épuisé / leurs marbres et leurs / bassins » (7). Les mots eux-mêmes deviennent le seul point d'appui. On sent le réel, sa présence, ses contours, mais en abîme ; tout cela est prêt à nous parler, à condition qu'on prête l'oreille.

Peu à peu se tissent des réseaux sonores. Dans ce cas, la consonne $[v]$ ajoute le sens d'un va-et-vient entre passé et présent, attente et action, au moyen de mots clés : «Villes », « [nous] avions », « Revint » (7); « Vignes », « vidaient», « recouvre» (8); « révèle», « ivoire», « veille» (9). À la fin du recueil, ces réseaux sonores nous aident à remarquer l'ouverture dont rêve le poète et à apercevoir d'autres rives. En particulier, comme nous avons pu le constater à propos du livre Le Troisième, la consonne sourde [f] - qui forme une paire minimale avec $[\mathrm{v}]$ - inscrit la durée dans l'étoffe langagière, en renforçant l'idée d'ouverture, de versants encore à imaginer et à juxtaposer. Sur le plan sémantique, les mots qui contiennent ces sons ajoutent une dimension très charnelle et féminine à la limpidité des ciels dans lesquels nous devenons « poreux », accueillons un « centre » et laissons « en suspens / / l'écume » des mots qui nous accompagne : «Bientôt s'efface / la frontière / de l'épaule / et des reins / un mouvement / frémit / respire / devient voile / rives s'inclinent / se font / contours » (154). À travers ce tissu sonore, le lecteur de Reverdy et de Du Bouchet sent encore une fois comment la poésie nous humanise tout au long de la vie, exigeant de nous rapprocher des réalités non pas à la va-vite, en hâtant le pas, mais en s'accordant à des fils langagiers secrets, subtils, inespérés.

Revenons donc aux «alliance[s]» (AR 21) dont parle Tellermann et au fait qu'elle emboîte le pas à ses pairs. Les traces reverdyennes que l'on peut distinguer dans ses recueils, bien que particulièrement révélatrices, ne sont qu'un signal qui nous est donné de nous consacrer nous-mêmes à des liens profonds avec « le bouche-abîme du réel désiré qui manque » qu'est la poésie. ${ }^{48}$ Comme les éléments constitutifs du réel semblent dispersés, d'autant que le discours ambiant s'en soucie peu, il faut tâcher de les réunir. C'est cela, la prière commune de Reverdy, de Du Bouchet et de Tellermann, leur réponse à l'énigme, leur dévoilement du mystère. Ils réactivent à travers le langage - et le dialogue avec soi et leurs semblables - la «matière-émotion $»^{49}$ des mondes intérieur et extérieur qui s'absente en grande partie du quotidien. Agir de la sorte est une clé de voûte de l'héritage littéraire que nous apporte

\footnotetext{
47 Cf. Jacques Darras, « L'énigme et la déception », op. cit.

48 Pierre Reverdy, En vrac [1956], OEuvres complètes II, op. cit., p. 941.

49 Michel Collot, La Matière-émotion, Paris : PUF, 1997.
} 
Reverdy, plutôt que de se dévouer à l'« avènement » des images. ${ }^{50}$ Enfin, on aperçoit d'autant mieux la promesse qu'est d'ores et déjà l'héritage tellermannien : celle de songer à partir de la langue de ses pairs, celle de s'allier intimement au langage concret de la réalité sensible.

50 André du Bouchet, «Envergure de Reverdy » [1951], Aveuglante ou banale : essais sur la poésie, 1949-1959, op. cit., p. 55. 